\title{
Dispersible Tablet for Dose Dispenser
}

National Cancer Institute

\section{Source}

National Cancer Institute. Dispersible Tablet for Dose Dispenser. NCI Thesaurus. Code C149438.

Solid preparation consisting of small, dispersible tablets that are designed to be used in a dose dispenser, each tablet usually consisting of a small fraction of a dose, with multiple tablets being automatically counted and administered as a single dose. 